\title{
Semi-analytical solutions for the delayed and diffusive viral infection model with logistic growth
}

\section{H. Y. Alfifi}

Department of Basic Sciences, College of Education, Imam Abdulrahman Bin Faisal University, Dammam, Saudi Arabia.

\begin{abstract}
In the one-dimensional reaction-diffusion domain of this study, semi-analytical solutions are used for a delayed viral infection system with logistic growth. Through an ordinary differential equations system, the Galerkin technique is believed to estimate the prevailing partial differential equations. In addition, Hopf bifurcation maps are constructed. The effect of diffusion coefficient stricture and delay on the model is comprehensively investigated, and the outcomes demonstrate that diffusion and delay can stabilize or destabilize the system. We found that, as the delay parameter values rise, the values of the Hopf bifurcations for growth and the rates of viral death are augmented, whereas the rate of production is decreased. For the growth, production, and death rates strictures, there is determination of an asymptotically unstable region and a stable region. Illustrations of the unstable and stable limit cycles, as well as the Hopf bifurcation points, are found to prove the formerly revealed outcomes in the Hopf bifurcation map. The results of the semi-analytical solutions and numerical assessments revealed that the semi-analytical solutions are highly effective.
\end{abstract}

Keywords: Reaction-diffusion, delay, Hopf bifurcations, viral infection, semi-analytical solutions, limit cycle. 2010 MSC: 35K57, 34C05, 34K18, 34-XX, 35-XX.

(C)2019 All rights reserved.

\section{Introduction}

For human health, biological applications are extremely significant and important. For a long time, these applications have been described and modeled by a number of practical researchers using partial differential equations (PDEs) or ordinary differential equations (ODEs). It is important that when these models consist of time delay and diffusion, that can be revealed in the model's dynamic behavior. In our everyday life, delayed reaction-diffusion models have revealed a number of oscillatory phenomena with the use of continuous well-stirred tank reactor (CSTR). CSTRs have obtained good results with experimental oscillatory systems in the theoretical research, for instance in logistical equations [4], the viral infection model [14, 20, 27], Nicholson's blowflies equation [5], and the limited food model [1].

A virus is a microscopic organism that can only replicate inside a host organism's cells, and they are among the most prevalent causes of ill health in humans. The majority of viruses are tiny, and cannot be

Email address: hyalfifi@iau.edu.sa (H. Y. Alfifi)

doi: $10.22436 /$ jnsa.012.09.04

Received: 2019-01-11 Revised: 2019-04-03 Accepted: 2019-04-07 
seen by unaided eyes, but can be observed using a conventional optical microscope. There are viruses that can infect all types of organisms, including animals, bacteria, plants, and archaea. Viruses are significant to human health, causing common illnesses as well as serious diseases. Mathematical epidemiology models can assist in understanding the viral transmission mechanism and how to control viral diseases $[15,23]$. A viral infection model with diffusion reaction and delay is investigated in this study.

Recently, Zhuang [27] considered a delay-diffusion model to investigate the spatiotemporal interactions between target cells, free virus molecules, and infected cells. He created a periodic phenomenon through diffusion stricture and spatial time delay and found that for any positive value of time delay, the healthy equilibrium is asymptotically stable when the rate of production stricture is small. In addition, the researcher presented a number of examples to affirm their analytical outcomes. In an additional study [14], a numeric technique was used for a time-delayed approach to the diffusive viral infection model, and the researchers applied the Lyapunov function, which exhibited equilibria equilibrium global stability of the conforming continuous model without limit on the space size or time. [24] proposed a simulated hepatitis B virus (HBV) infection with spatial dependence, where the perturbation technique was utilized in discovering the presence of traveling. Numerical results revealed that the model acknowledged traveling profiles that were non-monotone. In addition, they investigated the impact of a variety of strictures on the minimum wave speed. [13] investigated a delayed reaction-diffusion model of viral infection dynamics in vivo using the Lyapunov technique. [21] investigated the presence of HBV infection traveling wave solutions with the use of the upper-lower technique. In $[9,16,19,25,26]$, a number of diverse dynamic behaviors for the diffusive viral infection model with delay were considered.

Semi-analytical solutions have been applied to solve several complications with reaction-diffusion systems, such as predator-prey model [8], pellet systems [18], BZ reactions [6], the Brusselator system [2], mixed quadratic-cubic autocatalytic reactions [7], and logistic equations [3, 4]. All these models yielded accurate solutions compared to full numerical outcomes. Recently, Alfifi [2] introduced a Brusselator model semi-analytical system in the 1-D and 2-D domains. For the ODE system in that study to be attained, the Galerkin technique was used, and the Hopf bifurcation map, stability analysis, and steadystate solutions of the Hopf bifurcation points were constructed. The effects of diffusion strictures in the model were investigated, and the findings showed that an increase in the diffusion parameter in the model can have a stabilizing outcome. Further, Alfifi et al. [4] similarly investigated a class of generalized logistic PDEs with time delay inaugurated semi-analytical solutions, taking into consideration distributed and point delays for 1-D and 2-D domains. By using the Galerkin technique, an ODE system with delay was accomplished. For the stability analysis, the semi-analytical outcome was comprehensively presented.

The present study examined a viral infection model with delay in the 1-D reaction. In addition, the study has several objectives, such as illustrating the way the Galerkin averaging method is essential for ascertaining the way delay and diffusion strictures can affect the system and providing explanations about how computations can be brought into predicting the Hopf bifurcation points for the system and studying the stability. This paper is structured as follows. Section 2 explores the application of the Galerkin technique in driving ODE models. Section 3 explores the steady-state solutions that are connected to semi-analytical solutions (ODE system) and numerical solutions for PDEs. Section 4 discusses the theoretical framework for constructing the Bifurcation diagrams and Hopf bifurcation maps as well as illustrating several instances of limit cycles. A comparison of the semi-analytical and numerical models is also explored in each part.

\section{The semi-analytical model}

\subsection{Governing equations}

The non-linear system of delay PDEs with logistic growth and a reaction-diffusion viral infection model is expressed as follows:

$$
u_{t}=D u_{x x}+r u(t)[1-u(t)-w(t)]-u(t) v(t),
$$




$$
\begin{aligned}
w_{\mathrm{t}} & =D w_{x x}+u(t-\tau) v(t-\tau)-a w(t), \\
v_{\mathrm{t}} & =D v_{x x}+k w(t)-v \\
u_{x} & =w_{x}=v_{x}=0 \text { at } x=0 \\
u & =w=v=0, \text { at } x= \pm 1 \text { and } \mathrm{t}=0 \\
u & =u_{a}, w=w_{a}, v=v_{a}-\tau<t \leqslant 0
\end{aligned}
$$

whereby $w=w(x, t), u=u(x, t)$, and $v=v(x, t)$ refer to dimensionless concentrations of corresponding infected cells, uninfected cells, and free virus particles at time $t$ and location $x$, respectively. In the absence of virus and infected cells, the target cell's growth adheres to the logistic rule. This is a system that is open. At $x= \pm 1$, attached to a reservoir containing $u, w$, and $v$ at constant concentrations, the reactor has a permeable boundary. There is a zero flux boundary condition at the center of domain $x=0$. As a result, around the center of the concentrations $x=0$, the solution is symmetrical. The system comprises five positive strictures: $k>0$ represents the rate of production of free virus by infected cells, $D$ stands for the diffusion coefficient of the system, $a>0$ describes the rate of death in the infected cells, $\tau>0$ refers to the virus production lags after the infection, and $r>0$ is the rate of uninfected cell growth. Furthermore, $u_{a}, w_{a}$, and $v_{a}$ are considered to be the system's positive initial condition when $t$ ranges between $\tau$ and 0 .

The Runge-Kutta fourth-order technique [5, 18] and a Crank-Nicholson finite-difference scheme [4, 6] are introduced to the ODE and PDE numerical solutions and the spatial and temporal discretizations used in all the examples and figures are $\Delta x=0.05$ and $\Delta t=5 \times 10^{-3}$, respectively. The proportion of inaccuracy, which is considered to be the difference between the two-term semi-analytical solutions and the numerical solutions is defined in this paper as the unconditional value of the difference divided by the precise value times 100 .

\subsection{The Galerkin method}

With the use of the Galerkin technique, the semi-analytical system for (2.1) has been derived in the 1-D geometrical domains, and a spatial form of the profile concentration in this technique is taken into consideration $[2,4,17,18]$. The Galerkin technique refers to an analytical method that applies the orthogonality of a set of rudimentary roles to substitute PDEs with an ODE system. This technique has been applied in finding the solution for the model in many areas, for instance in neutron transport, structure mechanics, fluid flow, dynamics, and others [11]. The following trial function expansion is used:

$$
\begin{aligned}
& u(x, t)=u_{1}(t) \cos \left(\frac{\pi x}{2}\right)+u_{2}(t) \cos \left(\frac{3 \pi x}{2}\right), \\
& w(x, t)=w_{1}(t) \cos \left(\frac{\pi x}{2}\right)+w_{2}(t) \cos \left(\frac{3 \pi x}{2}\right), \\
& v(x, t)=v_{1}(t) \cos \left(\frac{\pi x}{2}\right)+v_{2}(t) \cos \left(\frac{3 \pi x}{2}\right) .
\end{aligned}
$$

The trial function is constructed such that $u=\sum u_{i}, w=\sum w_{i}$, and $v=\sum v_{i}$ refer to the concentrations at the center of the reaction diffusion cell. The trial function expansion (2.2) meets the boundary conditions labeled in $(2.1)$, for more details, see [3-5,17]. The free parameters in this expansion are therefore created through the evaluation of averaged versions of the governing equation weighted by the basic roles $\cos \left(\frac{1}{2} \pi x\right)$ and $\cos \left(\frac{3}{2} \pi x\right)$. Therefore, a system of presence of six ODEs as follows:

$$
\begin{aligned}
\frac{\mathrm{d} \mathfrak{u}_{1}}{\mathrm{dt}}= & -\frac{\pi^{2}}{4} \mathrm{D} \mathfrak{u}_{1}+r \mathfrak{u}_{1}-\frac{8}{3 \pi} r u_{1}^{2}-\frac{16}{15 \pi} r u_{1} u_{2}-\frac{8}{3 \pi} r u_{1} w_{1}-\frac{8}{15 \pi} r u_{1} w_{2}-\frac{72}{35 \pi} r u_{2}^{2} \\
& -\frac{8}{15 \pi} r u_{2} w_{1}-\frac{72}{35 \pi} r u_{2} w_{2}-\frac{8}{3 \pi} u_{1} v_{1}-\frac{8}{15 \pi} \mathfrak{u}_{1} v_{2}-\frac{8}{15 \pi} \mathfrak{u}_{2} v_{1}-\frac{72}{35 \pi} \mathfrak{u}_{2} v_{2}, \\
\frac{\mathrm{d} w_{1}}{\mathrm{dt}}= & -\frac{\pi^{2}}{4} D w_{1}-w_{1} \mathrm{a}+\frac{8}{3 \pi} \mathfrak{u}_{1 \mathrm{~d}} v_{1 \mathrm{~d}}+\frac{8}{15 \pi} \mathfrak{u}_{1 \mathrm{~d}} v_{2 \mathrm{~d}}+\frac{8}{15 \pi} \mathfrak{u}_{2 \mathrm{~d}} v_{1 \mathrm{~d}}+\frac{72}{35 \pi} \mathfrak{u}_{2 \mathrm{~d}} v_{2 \mathrm{~d}},
\end{aligned}
$$




$$
\begin{aligned}
& \frac{\mathrm{d} v_{1}}{\mathrm{dt}}=-\frac{\pi^{2}}{4} \mathrm{D} v_{1}+w_{1} \mathrm{k}-v_{1} \\
& \frac{d u_{2}}{d t}=-\frac{9 \pi^{2}}{4} D u_{2}+r u_{2}-\frac{8}{15 \pi} r u_{1}^{2}-\frac{144}{35 \pi} r u_{1} u_{2}-\frac{8}{15 \pi} r u_{1} w_{1}-\frac{72}{35 \pi} r u_{1} w_{2}+\frac{8}{9 \pi} r u_{2}^{2} \\
& -\frac{72}{35 \pi} \mathrm{ru}_{2} w_{1}+\frac{8}{9 \pi} \mathrm{ru}_{2} \boldsymbol{w}_{2}-\frac{8}{15 \pi} \mathfrak{u}_{1} v_{1}-\frac{72}{35 \pi} \mathfrak{u}_{1} v_{2}-\frac{72}{35 \pi} \mathfrak{u}_{2} v_{1}+\frac{8}{9 \pi} \mathfrak{u}_{2} v_{2} \\
& \frac{\mathrm{d} w_{2}}{\mathrm{dt}}=-\frac{9 \pi^{2}}{4} \mathrm{D} w_{2}-w_{2} \mathrm{a}+\frac{8}{15 \pi} \mathfrak{u}_{1 \mathrm{~d}} v_{1 \mathrm{~d}}+\frac{72}{35 \pi} \mathfrak{u}_{1 \mathrm{~d}} v_{2 \mathrm{~d}}+\frac{72}{35 \pi} \mathfrak{u}_{2 \mathrm{~d}} v_{1 \mathrm{~d}}-\frac{8}{9 \pi} \mathfrak{u}_{2 \mathrm{~d}} v_{2 \mathrm{~d}} \text {, } \\
& \frac{\mathrm{d} v_{2}}{\mathrm{dt}}=-\frac{9 \pi^{2}}{4} \mathrm{D} v_{2}+w_{2} \mathrm{k}-v_{2} \\
& u_{i d}=u_{i}(x, t-\tau), \quad v_{i d}=v_{i}(x, t-\tau), \quad i=1,2 .
\end{aligned}
$$

After finding two terms in (2.2), the series are abbreviated, and the number of terms utilized in the truncated series stand for a trade-off between the complexity and the accuracy of the semi-analytical solution. It is manifestly exposed that abundant accuracy with little expression swell is delivered by the two-term technique. The system (2.3) comprises six diverse ODEs, because (2.2) consists of three diverse trial function terms. In particular, the one-term solutions are generated a through setting of $u_{2}=w_{2}=v_{2}=0$.

\section{Positive steady-state bifurcations}

For the steady-state solutions to be obtained in (2.3), let $u(t)=u_{s}=u(t-\tau)$ and $v(t)=v_{s}=v(t-\tau)$, which are reduced to the sets of transcendental equations. Therefore, the Maple software package is applied in acquiring the steady-state solutions through the solving of the transcendental equations $\frac{d u_{i}}{d t}=$ $\frac{\mathrm{d} w_{i}}{\mathrm{~d} t}=\frac{\mathrm{d} v_{i}}{\mathrm{~d} t}=0, i=1,2$.

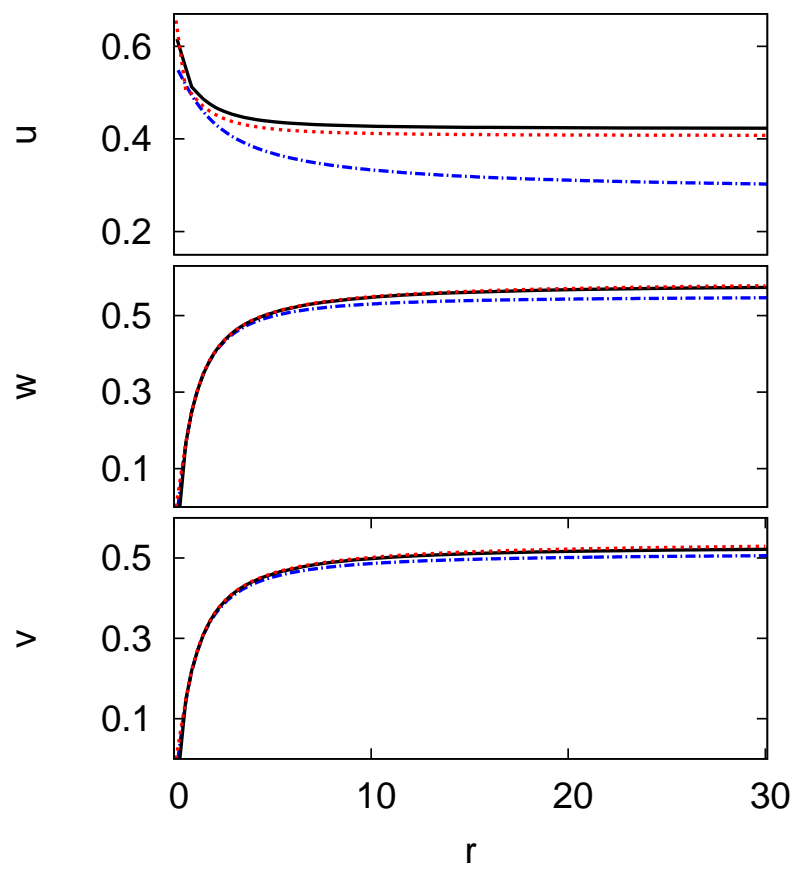

Figure 1: (color online) The steady-state reactant concentrations $u, v$ and $w$ versus $r$ at $x=0$. The solid black line and dashed blue lines stand for the two-term and one-term semi-analytical solutions and the dotted red line symbolizes the specification of (2.1) applied for PDEs. The parameters spaces are $\mathrm{k}=1, \mathrm{a}=0.3, \mathrm{D}=0.05$, and $\mathrm{u}_{\mathrm{a}}=v_{\mathrm{a}}=w_{\mathrm{a}}=0.1$.

In Figure 1, the steady-state reactant concentrations $w, v$, and $u$ versus uninfected cell growth rate $r$, is clearly illustrated, and the parameter spaces are $\mathrm{k}=1, \mathrm{a}=0.3, \mathrm{D}=0.05$, and $\mathrm{u}_{\mathrm{a}}=v_{\mathrm{a}}=w_{\mathrm{a}}=0.1$. This figure shows both the one- and two-term semi-analytical models (blue, black) along with numerical 
solutions of the PDEs (red line) displayed at the domain center, $x=0$. In every reactant of concentrations $v, u$, and $w$, there is a unique pattern of steady-state solutions. The figure clearly illustrates that as $r$ increases, $u$ decreases before a minimum at a large value of $r$ is approached. However, the curves $v$ and $w$ linearly increase as uninfected target cells growth rate $r$ increases. There is an outstanding comparison that exists between numerical solutions and the two-term semi-analytical solution, with not more than $2 \%$ error for all values of growth rate $r$ over the selected domain of up to $r=30$. For the concentrations $v$ and $w$, the difference between the one-term semi-analytical solution and the numerical solution is rationally precise, whereas over the domain, the concentration $u$ is somewhat higher.

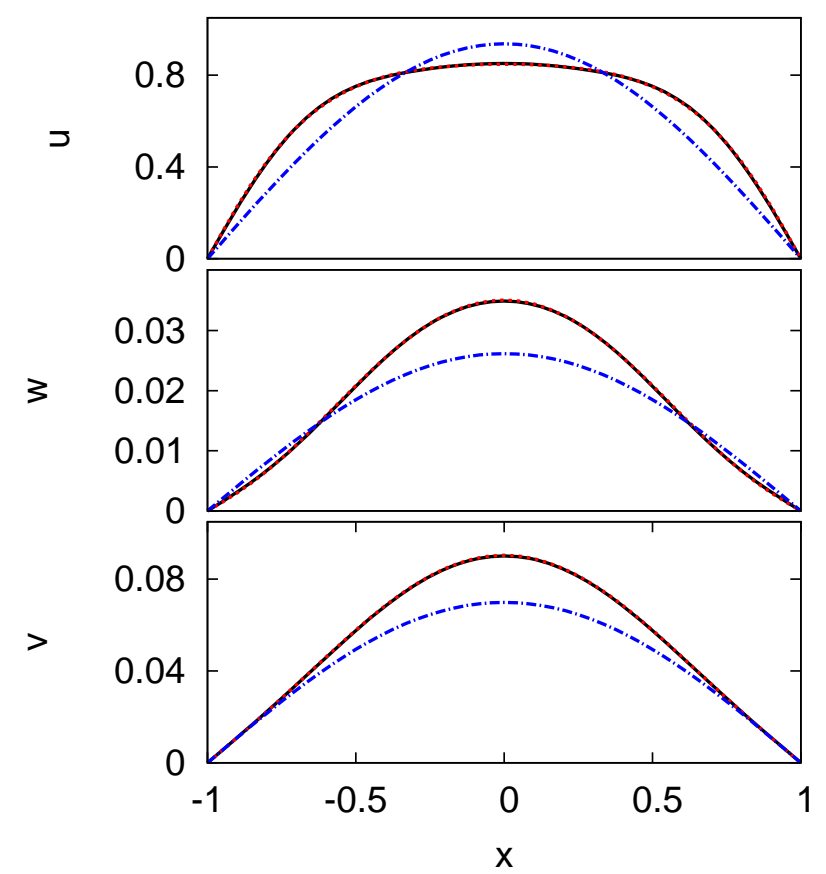

Figure 2: (color online) The steady-state reactant concentrations $u, v$, and $w$ against $x$. The parameters used are $r=1, a=2, k=3$, $\mathrm{D}=0.05$, and $\mathrm{u}_{\mathrm{a}}=v_{\mathrm{a}}=w_{\mathrm{a}}=0.1$. The solid black line and the dashed blue line stand for two-term and one-term semianalytical solutions, and the dotted red line symbolizes the specification applied for the PDEs of (2.1).

Figure 2, shows the steady-state reactant concentrations $w, v$, and $u$ against $x$. It is an illustration of the one-term and two-term semi-analytical solutions as well as the numerical solution of the governing PDEs. The applied parameters are $r=1, a=2, k=3, D=0.05$, and $u_{a}=v_{a}=w_{a}=0.1$. For the reactant concentrations $u, w$, and $v$, the solutions have a single hump; all solutions are offered. It is obvious from the figures that at $x=0$, the one-term peak solution is $u=0.929$. The two-term solution has a density of $u=0.850$ at $x=0$, which is very close to the numerical density of $u=0.848$. For the curve $w$, the numerical solution at $x=0$ is $w=0.035$, while the two- and one-term solutions at $x=0$ are $w=0.035$ and 0.028 , respectively. Moreover, in the curve $v$, the one-term peak solution at $x=0$ is $v=0.072$. The two-term solution has a density of $v=0.090$ at $x=0$, which is the same as a numerical density of $v=0.090$. It can be clearly observed that the comparisons between the two-term semi-analytical solutions and numerical solutions of PDEs are ideal across the domain, with an error rate of less than $0.5 \%$. The one-term estimate is reasonably accurate at the domain center $x=0$. In all domain regions, the two-term solution proved to be an exceptional model because it better estimates the solutions profiles.

\section{Stability analysis and Hopf bifurcations}

\subsection{Theoretical framework}

The technique applied in assessing the stability and Hopf bifurcation points of the semi-analytical system (2.3) are described in this section. A Hopf bifurcation signifies the periodic solutions appearance in 
the neighborhood of a steady state whose stability changes as a result of a conjugated pair of eigenvalues that cross over the imaginary axis [22]. A number of texts on bifurcation the theory and dynamical systems describe theory of Hopf bifurcations for ODE systems; for more details, see [10, 12].

Hopf points here are discovered through expansion in a Taylor series around the steady-state solution, as shown in equation (4.1):

$$
u_{i}=u_{i s}+\epsilon c_{i} e^{-m t}, \quad w_{i}=v_{i s}+\epsilon g_{i} e^{-m t}, \quad v_{i}=v_{i s}+\epsilon z_{i} e^{-m t}, \quad \text { where } i=1,2, \quad \text { ffl } \ll 1 .
$$

Therefore, equations (4.1) are substituted into ODEs system as illustrated in equation (2.3), in addition to being linearized around the steady state. Remarkably, Jacobian matrix eigenvalues reconnoiter the small system perturbation development, which illustrates the typical equation of the rate of growth $\mathrm{m}$. We let $m=i w$ in the characteristic equation, and the real part $(R)$ is separated together with the imaginary part (I). The Hopf bifurcation points are derived through solving the following equations:

$$
R=I=\frac{d u_{i}}{d t}=\frac{d w_{i}}{d t}=\frac{d v_{i}}{d t}=0, \quad i=1,2,
$$

where $\frac{d u_{i}}{d t}, \frac{d v_{i}}{d t}$, and $\frac{d w_{i}}{d t}$ refer to the steady-state system of (2.3).

\subsection{Hopf bifurcation regions}

A semi-analytical map in which the Hopf bifurcations take place is derived and contrasted with numerical outcomes. In addition, the effects of delay stricture and diffusion coefficients on the model are investigated with a number of numerical illustrations, all of which will be discussed in this section. Note that, in this section, the parameters $\mathrm{D}=0.05$ and $\mathrm{u}_{\mathrm{a}}=v_{\mathrm{a}}=w_{\mathrm{a}}=0.1$ are applied in all figures.

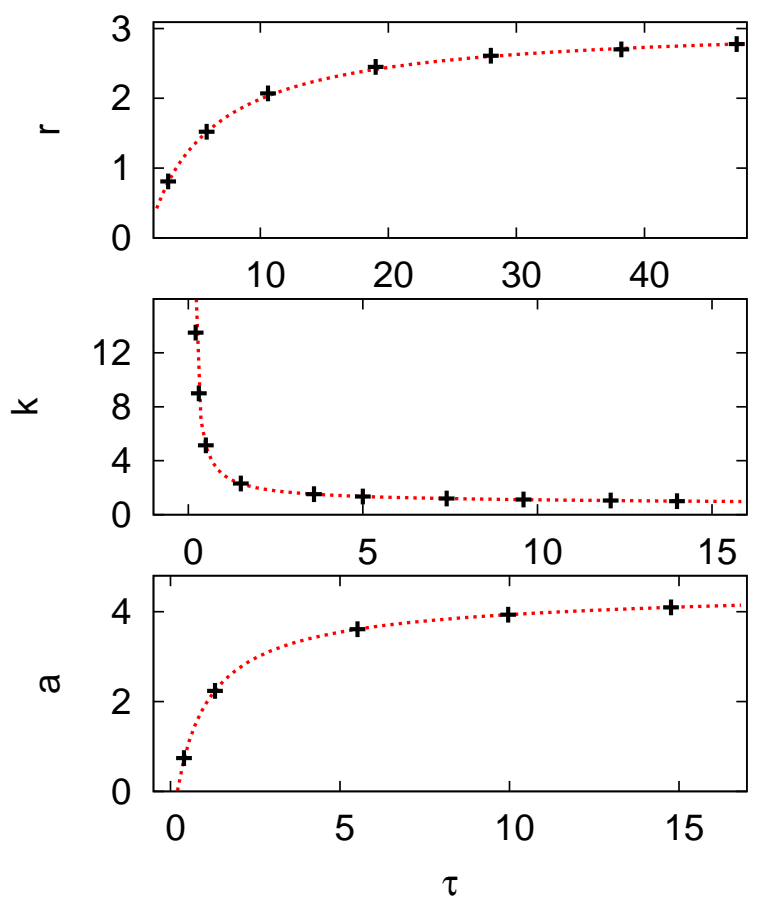

Figure 3: (color online) Curves of Hopf bifurcation for delay parameter $\tau$ against $r, k$, and a. The two-term semi-analytical solution is described by dotted red line while black crosses shows a numerical solution of the PDEs (2.1). The parameters are $k=1$ and $a=0.3$ in the $\tau-r$ plane (upper figure), $r=5$ and $a=0.3$ in the $\tau-k$ plane (middle figure), and $r=5$ and $k=10$ in the $\tau-$ a plane (lower figure).

Figure 3 illustrates the curves of the Hopf bifurcation in $\tau$ against the parameters $r$ (upper figure), $k$ (middle figure), and a (lower figure). The two-term semi-analytical solution (dotted red line) and numerical solution (black crosses) have been clearly shown. The parameters are $k=1$ and $a=0.3$ in the 
$\tau-r$ plane (upper figure), $r=5$ and $a=0.3$ in the $\tau-k$ plane (middle figure), and $r=5$ and $k=10$ in the $\tau-a$ plane (lower figure). These figures are illustrations that when there is an increase in the delay strictures $\tau$, the values that are critical in Hopf bifurcations for the uninfected cell growth rate $r$ and the rate of death a are augmented. However, the curve for the production rate of free virus $k$ is decreased as the delay parameter $\tau$ increased; this outcome is the same as that previously reported in [27]. It is clear that the two predictions pertaining to two-term semi-analytical solutions correspond with the numerical calculations with an error rate that does not exceed $2 \%$ for all choices.

Figure 4 illustrates the Hopf bifurcation curves in the $k-r$ diagram. The two-term semi-analytical solution and numerical solution are drawn. The parameters applied are $\tau=1$ and $a=0.3$. The stability curve for numerical solutions and the two-term semi-analytical ODE systems divides the stricture space into two sections: the upper region, which is unstable, and the lower region which is stable. It is well illustrated in Figure 4 that the rate of production of free virus $k$ upsurges, and the critical value of growth rate $r$, whereby Hopf bifurcations first take place, decreases. Further, it can be clearly noted that the predictions pertaining to the two-term semi-analytical solutions correspond with the numerical calculations with an error rate that does not exceed 3\% for all $r$ selections.

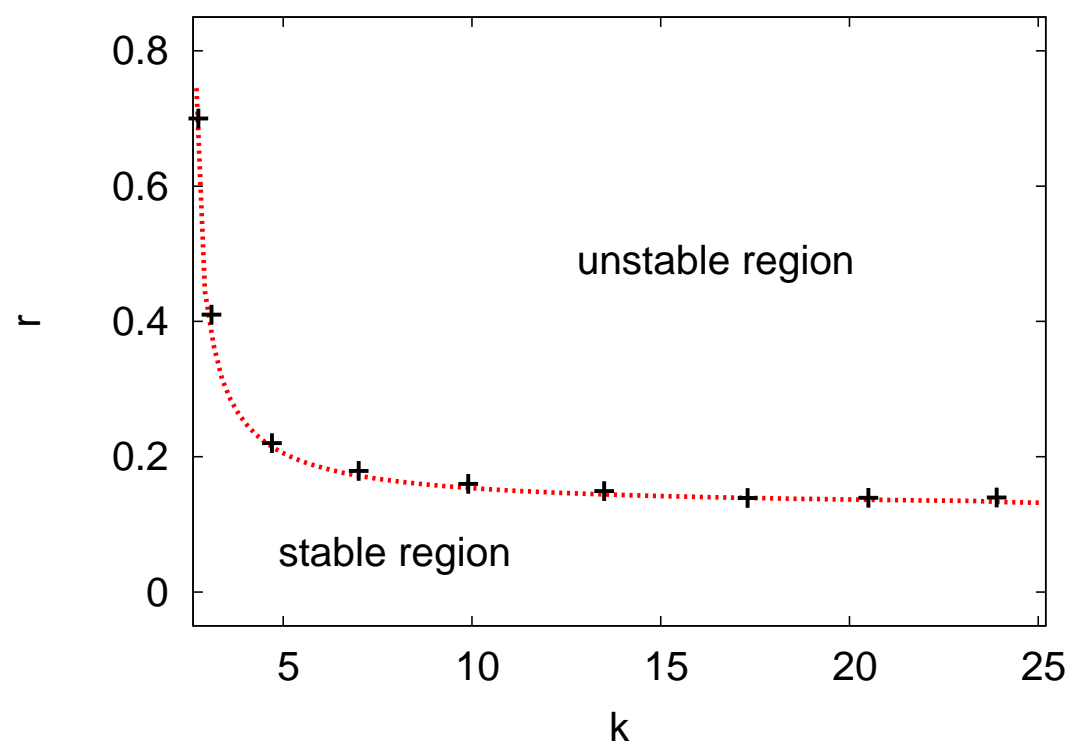

Figure 4: (color online) Curves of Hopf bifurcation k-r map. The two-term semi-analytical solution (dotted red line) and the numerical solution of the PDEs (black crosses) are shown. The parameters used are $\tau=1$ and $a=0.3$.

In Figure 5, the Hopf bifurcation curves in the k-r map for the diverse diffusion coefficient values are illustrated. Two-term semi-analytical solutions are derived for six diverse values of diffusion, namely $0.1,0.06,0.05,0.04,0.03$, and 0 . It can be seen that appropriately selected values of the diffusion of the stricture D can destabilize or stabilize regions of stricture space. It is also clear that, for any fixed value of the rate of production $k$ (given $r$ ), the system is destabilized as diffusion parameter D increases, when the critical value of growth rate $r$ is augmented. However, for all the diverse instances shown in Figure 5 , as the growth rate $r$ rises, the critical value of the production rate $k$ is decreased. It can therefore be determined that the diffusion stricture D can influence and stand for a destabilized or stabilized system.

Figure 6 illustrates curves of Hopf bifurcation in the diffusion coefficient D versus the growth rate $r$ (upper figure), the production rate $k$ (middle figure), and the death rate a (lower figure). In each figure, the two-term semi-analytical solution is displayed. The figures illustrate the occurrence of an upsurge in the diffusion coefficient $D$; the values that are essential in Hopf bifurcations for the rate of growth $r$ as well as the rate of production $k$ are augmented. As diffusion coefficient $\mathrm{D}$ augmented, however, the curve is decreased for the rate of death of free virus a. It can therefore be resolved that the diffusion parameter D can play a very crucial effect in the model and can denote a system that is destabilized or stabilized. 


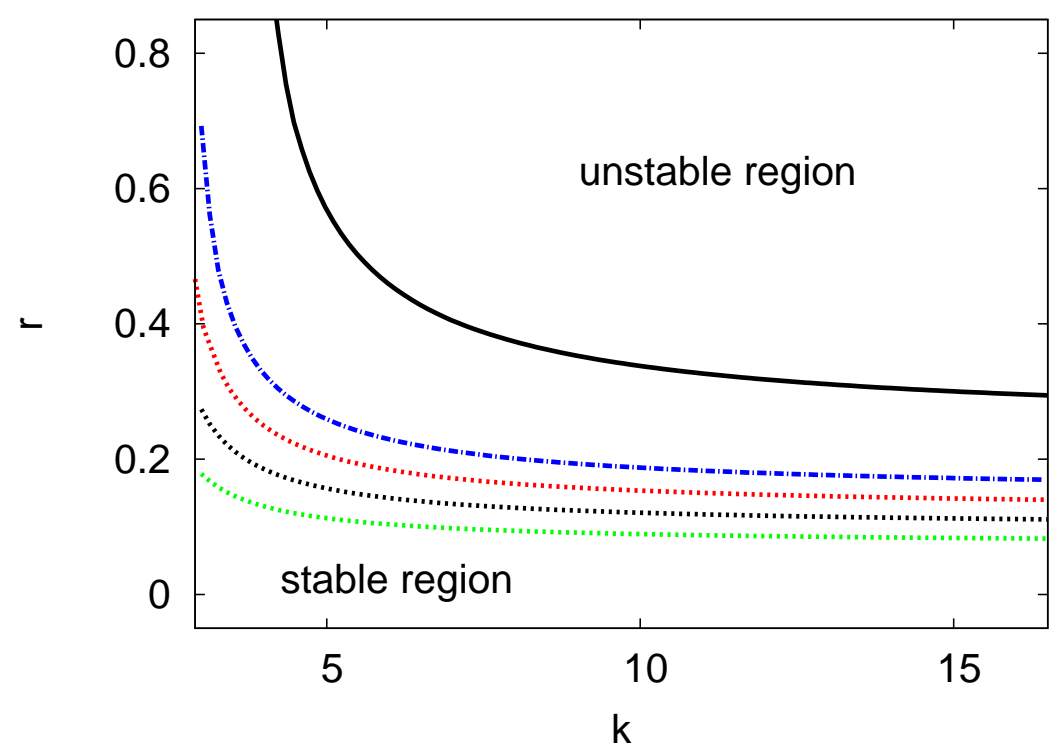

Figure 5: (color online) Hopf bifurcation curves in the $k-r$ plane, for different values of $D$, as follows: $D=0.1$ (solid black line), $\mathrm{D}=0.06$ (dashed blue line), $\mathrm{D}=0.05$ (dotted red line), $\mathrm{D}=0.04$ (dotted black line), and $\mathrm{D}=0.03$ (dotted green line). The two-term semi-analytical solution of (2.3) is illustrated. The parameters applied are $\tau=1$ and $a=0.3$.

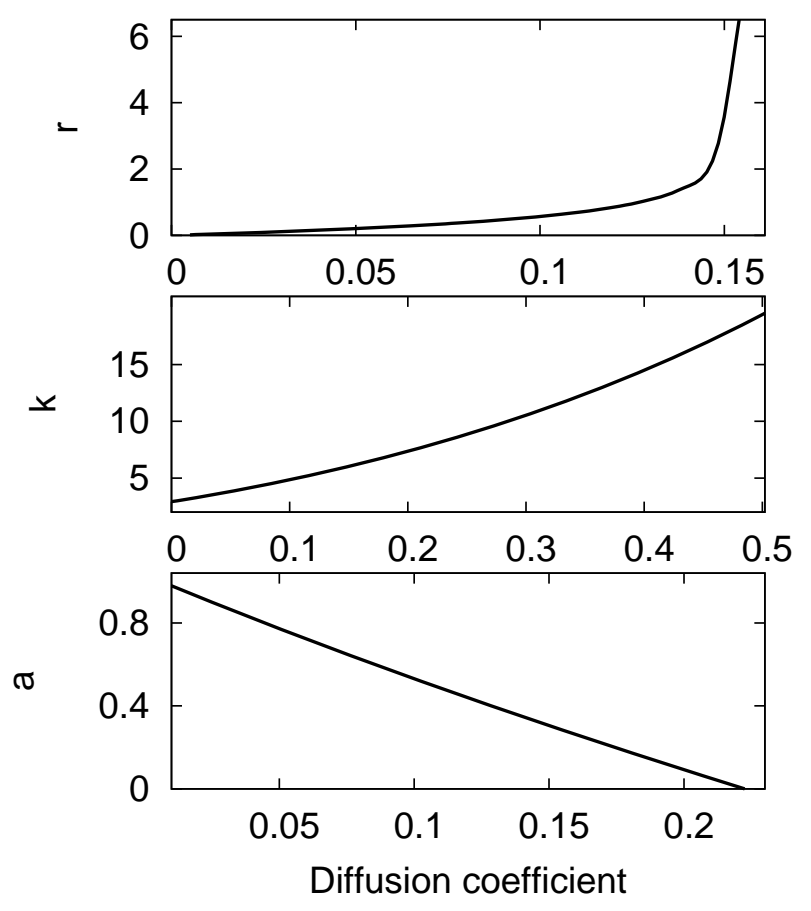

Figure 6: Curves of the Hopf bifurcation for diffusion coefficient D against $r, k$, and $a$. In each case, the two-term semi-analytical solution is presented. The parameters are $k=5$ and $a=0.3$ in the $D-r$ map (upper figure), $r=5$ and $a=0.3$ in the $D-k$ map (middle figure), and $r=k=5$ in the $D-a$ map (lower figure).

Figures 7 (a) and (b) display the Hopf bifurcation curves for the death rate parameters a versus the growth rate $r$ (left figure) and the production rate $k$ (right figure). The parameters are $r=10$ and $\tau=1$ for Figure 7 (a) and $k=5$ and $\tau=1$ for Figure 7 (b). The two-term semi-analytical solution is illustrated. In both cases, as the Hopf bifurcation of the rate of growth $r$ and the rate of production $k$ rises and the death rate strictures $a$ is increased.

We also displayed a number of comparisons for the special case where the parameters are $k=1$, 


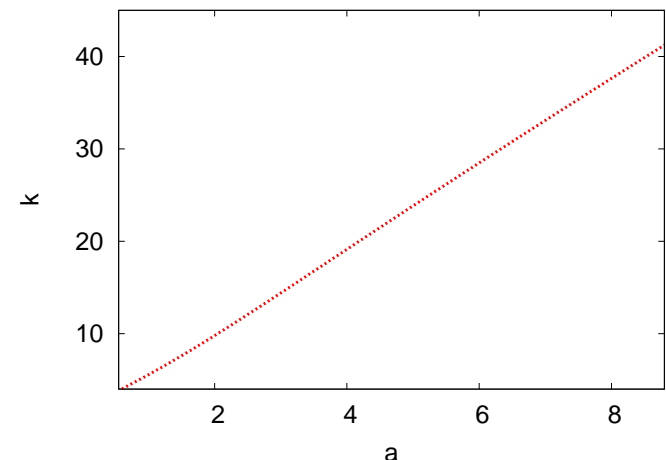

(a)

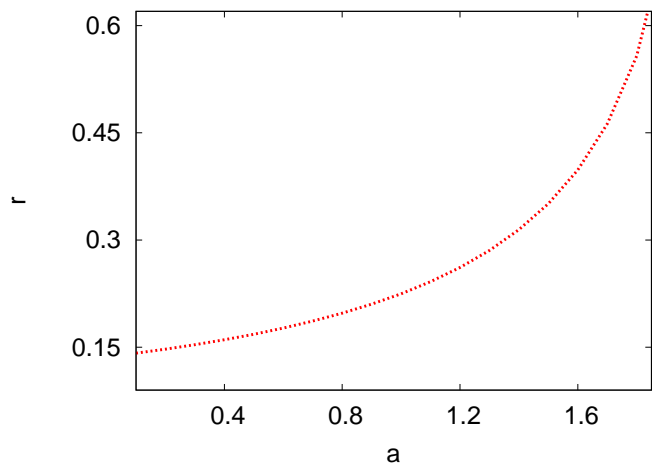

(b)

Figure 7: (color online) Curves of Hopf bifurcation in the $\mathrm{a}-\mathrm{k}$ plane for (a) and $\mathrm{a}-\mathrm{r}$ plane for (b). The two-term semi-analytical solution with $r=10$ and $\tau=1$ for Figure (a) and $k=5$ and $\tau=1$ for Figure (b) are shown.

$\tau=10, \mathrm{a}=0.3$, and $\mathrm{D}=0.05$. Here, the Hopf bifurcation points obtained are $\mathrm{r}_{\mathrm{c}} \simeq 1.98,2.02$, and 2.04 for the one-, two-term semi-analytical, and numerical solutions, respectively. The predictions of the semi-analytical theory (two-term) agreed and are closer to the numerical predictions, with less than a $1 \%$ difference. The semi-analytical solution is therefore, an outstanding predictor of the Hopf bifurcation incidence.

\subsection{Bifurcation diagrams and limit cycles}

The evolution and bifurcation diagrams for both limit cycles and steady-state solutions are discussed in this section. It should be noted that the bifurcation diagrams show long-time solutions of the steadystate amplitude as well as the minimum and maximum of the periodic oscillations amplitudes, so they are not functions of the initial condition $u_{a}, w_{a}, v_{a}$. In addition, the bifurcation diagrams illustrate the populations at the domain center $x=0$.

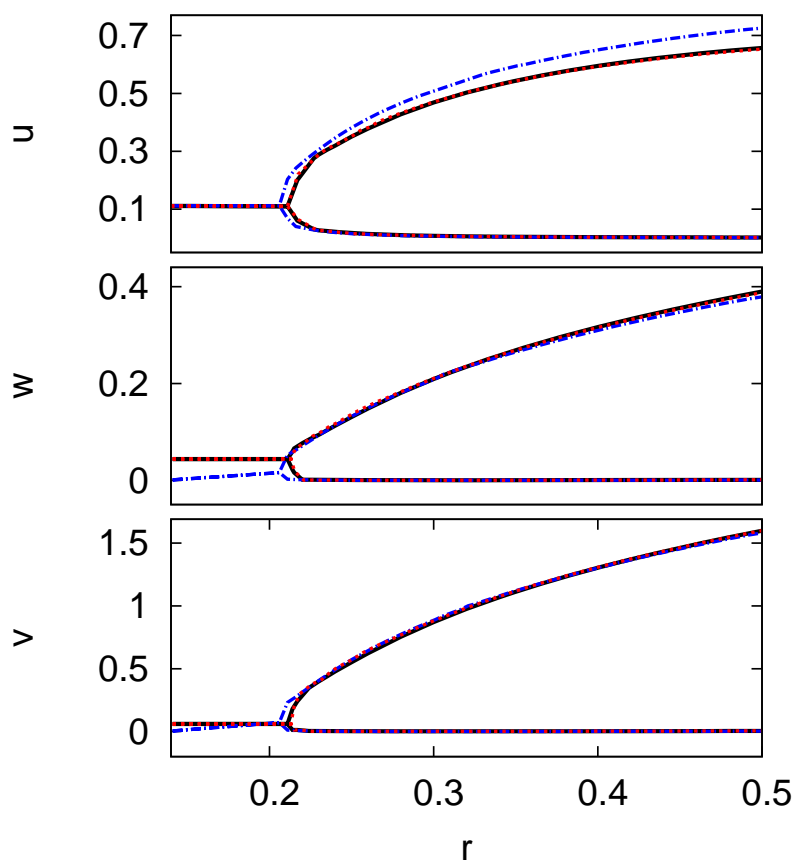

Figure 8: (color online) Bifurcation diagram of the reactant concentrations $w, v$, and $u$ versus $r$. The two-term (solid black line) and one-term (dashed blue line) semi-analytical solutions as well as the numerical solution of PDEs (dotted red line) are shown. The parameters are $\tau=1, k=5, \mathrm{D}=0.05, \mathrm{a}=0.3$, and $\mathrm{u}_{\mathrm{a}}=v_{\mathrm{a}}=w_{\mathrm{a}}=0.1$. 
Figure 8 shows the bifurcation diagram of the reactant concentrations $w, v$, and $u$ versus the uninfected cell growth rate $r$. This figure can be also explain how closely relate between numerical and semi-analytical solutions. The two and one-term semi-analytical solutions and the numerical solution are illustrated. The parameters that are applied in this analysis are; $\tau=1, k=5, D=0.05, a=0.3$, and $u_{a}=v_{a}=w_{a}=0.1$. The steady-state solutions are stable in every situation at $r<r_{c} \simeq 0.21$. Following the point, there is an immediate incident of the periodic solutions (supercritical Hopf bifurcation). The one and two-term semi-analytical points of Hopf bifurcation are $r_{c}=0.206$ and 0.213 , respectively. Whereas, the Hopf bifurcation numerical point is at $r_{c}=0.215$. It can be seen that as $r$ increases, the maximum amplitudes during oscillations also increases. The minimum amplitudes remain low, and thus, the predictions pertaining to the two semi-analytical solutions correspond with the numerical predictions. In this situation, there is an error rate of not more than $1 \%$ for the remaining $r$ choices.

Figures 9 and 10 the reactant concentrations $w, v$ and $u$ at center of domain $x=0$ versus time $t$ are illustrated. The parameters are $u_{a}=v_{a}=w_{a}=0.1, k=5, a=0.3$, and $\tau=1$, with $r=0.15$ for Figure 9 (from stable region shown in Figure 4) and $r=0.26$ for Figure 10 (in the unstable region of Figure 4). For every concentration, the one-term and two-term solutions, along with the numerical solution are clearly illustrated. For Figure $9 r=0.15<r_{c}$ and the solution evolves to a steady state with $\mathrm{u}(0, \mathrm{t}) \simeq 0.111, w(0, \mathrm{t}) \simeq 0.015$, and $v(0, \mathrm{t}) \simeq 0.003$ as the time $\mathrm{t}$ becomes large, following some original relaxation oscillations. The comparison between the two-term semi-analytical and numerical solutions is excellent, with only a $0.1 \%$ error rate at the steady state. The difference between the one-term semianalytical solution and the numerical solution is also excellent, with an error rate of less than $1 \%$. For Figure 10, $r=0.26>r_{c}$, and periodic solutions occur in the regions where the solutions loss stability. The numerical amplitude of the limit cycle for the reactant concentrations $u, w$, and $v$ are $0.277,0.091$, and 0.385 , respectively. These values are very close to the two-term semi-analytical amplitudes of $0.271,0.088$, and 0.370 , respectively. In addition, the difference between the two-term semi-analytical values and the numerical solutions are below $4 \%$. These figures confirmed the outcome obtained in Figure 8 .

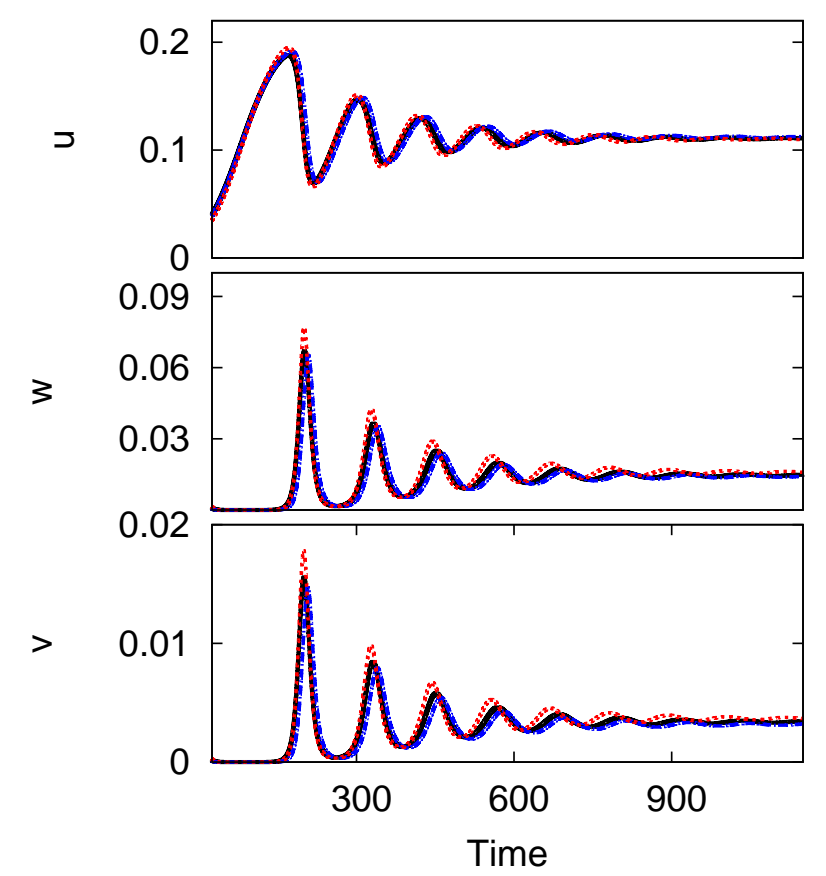

Figure 9: (color online) The reactant concentrations $w, v$, and $u$ at $x=0$ versus time. The two-term (solid black line) and oneterm (dashed blue line) semi-analytical solutions and the numerical solution (dotted red line) are illustrated. The parameters are $\tau=1, k=5, \mathrm{D}=0.05, \mathrm{a}=0.3, \mathrm{u}_{\mathrm{a}}=v_{\mathrm{a}}=w_{\mathrm{a}}=0.1$, and $\mathrm{r}=0.15$. 


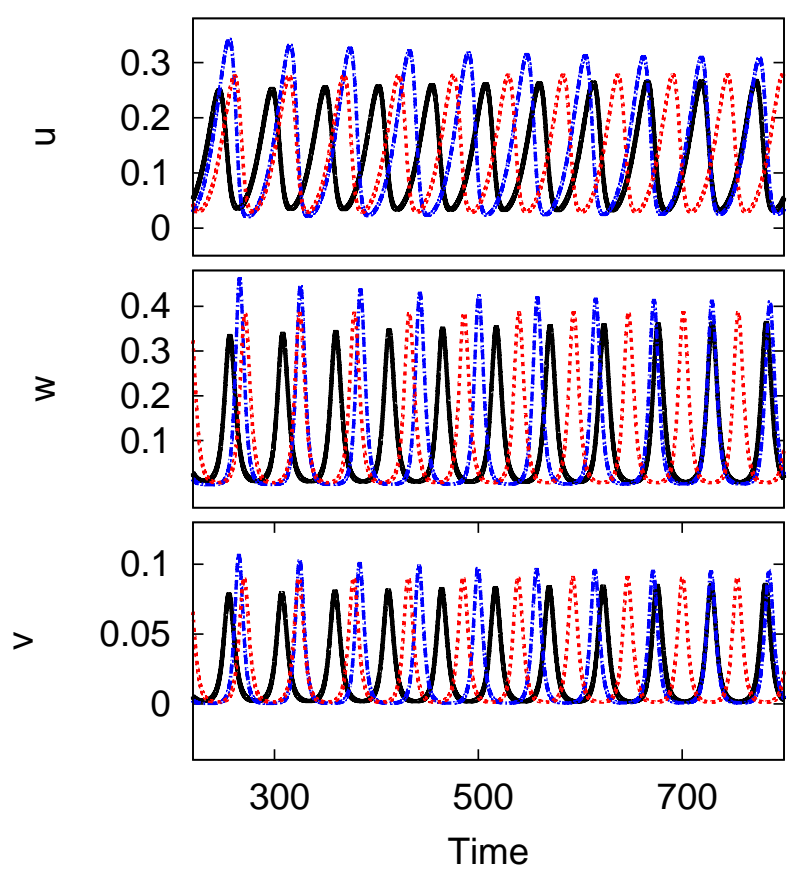

Figure 10: (color online) The reactant concentrations $w, v$, and $u$ at $x=0$ versus time. The two-term (solid black line) and oneterm (dashed blue line) semi-analytical solutions as well as the numerical solution (dotted red line) are shown. The parameters are $\tau=1, k=5, D=0.05, a=0.3, u_{a}=v_{a}=w_{a}=0.1$, and $r=0.26$.

Figure 11 the limit cycle curves in a view of the 3-D phase space for the reactant concentrations $u$ versus $w$ versus $v$ are drawn. The numerical solution for the PDEs and the one-term and the two-term semi-analytical solutions are illustrated. The parameter spaces applied are $\tau=1, k=5, D=0.05, a=0.3$, $u_{a}=v_{a}=w_{a}=0.1$, and $r=0.26$. For the reactant concentrations $u, w$, and $v$, the numerical period of the limit cycle is 53.8, whereas the two-term and one-term semi-analytical are 53.6 and 56.2, respectively. The difference between the two-term semi analytical and the numerical solutions is quite good, with only a $1 \%$ error rate. The two-term semi-analytical approximation is somewhat adjacent to the numerical solution over the entire stricture space, and the semi-analytical limit cycle has numerous quantitative relationships with the numerical solution.

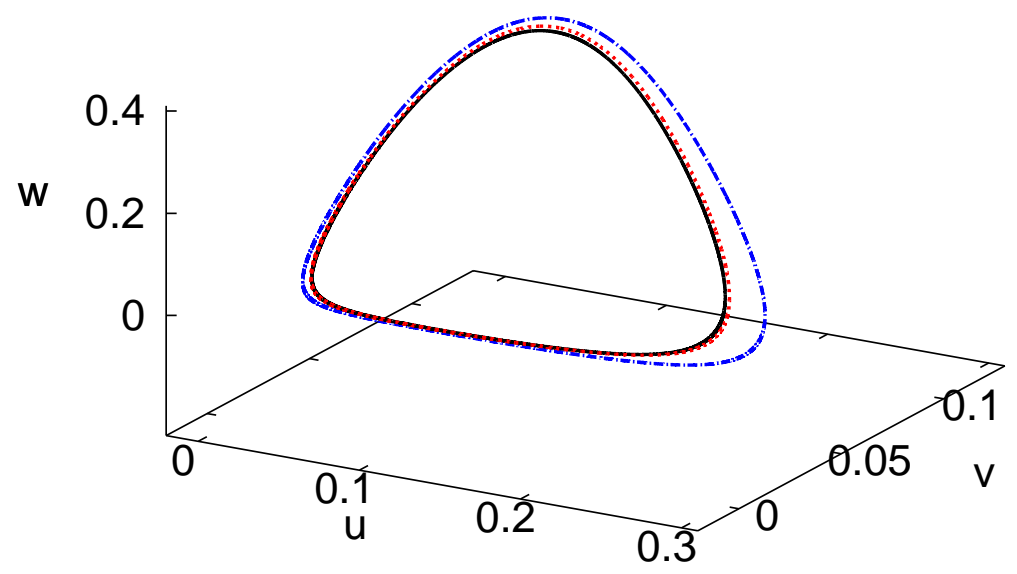

Figure 11: (color online) The reactant concentrations $u, w$, and $v$ at $x=0$ versus time $t$. The two-term (solid black line) and one-term (dashed blue line) semi-analytical solutions as well as the numerical solution (dotted red line) are illustrated. The parameters are $\tau=1, k=5, \mathrm{D}=0.05, \mathrm{a}=0.3, \mathrm{u}_{\mathrm{a}}=v_{\mathrm{a}}=w_{\mathrm{a}}=0.1$, and $\mathrm{r}=0.26$. 
Figure 12 shows the 3-D phase space of the limit cycle for four different values of growth rate $r$ : $r=0.5$ (dotted green), $r=1$ (dotted blue), $r=1.5$ (dotted black), and $r=2$ (dotted red). The twoterm semi-analytical solution is drown. The parameters used are $\tau=1, k=5, D=0.05, a=0.3$, and $u_{a}=v_{a}=w_{a}=0.1$. This figure is an illustration of the nature of the limit cycles for greater values of growth rate $r$, well beyond the Hopf bifurcation point. Many biological systems, such as the Nicholson's blowflies equation (see Alfifi et al. [5]) display period doubling and chaotic solutions. This behavior does not occur for the delayed viral infection ODE or PDE systems. This figure also indicates that the oscillations amplitudes continue increasing as $r$ similarly upsurges and that the limit cycle curve takes a shape of a triangle.

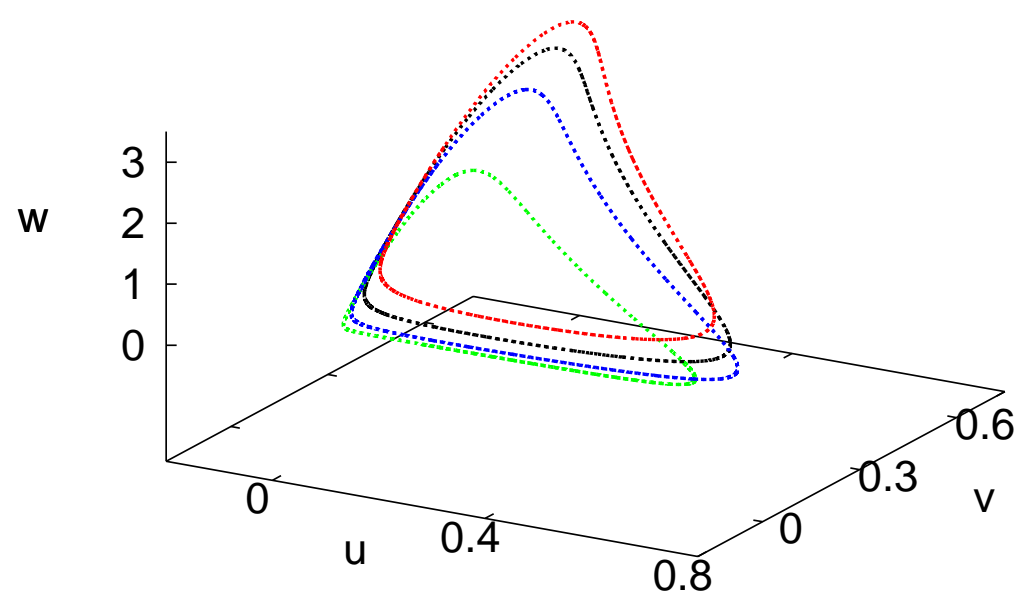

Figure 12: (color online) The limit cycle for four different values of $r: r=0.5$ (dotted green line), $r=1$ (dotted blue line), $r=1.5$ (dotted black line), and $r=2$ (dotted red line). The two-term semi-analytical solution is illustrated. The parameters are used $\tau=1, k=5, D=0.05, a=0.3$, and $u_{a}=v_{a}=w_{a}=0.1$.

\section{Conclusions}

This paper presented the viral infection system's lower-order semi-analytical model with delay in the 1-D domain. The Galerkin technique was applied in producing an ODE system. We successfully discovered the system's steady-state solutions, constructed a Hopf bifurcation diagram, and carried out an analysis of the stability (Hopf bifurcation points). A Hopf bifurcations map was shown, and the outcomes of delay and diffusion parameters in the model were investigated. We found that the effects of the delay coefficients on the system can destabilize or stabilize parameter space regions, but within the system, the rise in diffusion coefficients can have a stabilizing outcome, such that the diffusion stricture increased. Further, the critical values of the Hopf bifurcation point were increased for the rate of growth and production, whereas the free virus rate of death was reduced. Examples of unstable and stable limit cycles were also presented. We discovered that there were no chaotic solutions or display period doubling over long periods of time. Moreover, relationships between the PDE systems (numerical solutions) and the semi-analytical (two-term) solutions revealed the efficacy of the semi-analytical method. Ultimately, these outcomes reveal that the semi-analytical technique is beneficial and offers a precise analytical technique for PDE systems evaluation. In future studies, we will focus on using this technique with a different system of delayed reaction-diffusion cell.

\section{References}

[1] H. Y. Alfifi, Semi-analytical solutions for the delayed diffusive food-limited model, 7th International Conference on Modeling, Simulation, and Applied Optimization ICMSAO (Sharjah, U. A. E.), 2017 (2017), 1-5. 1 
[2] H. Y. Alfifi, Semi-analytical solutions for the Brusselator reaction-diffusion model, 59 (2017), ANZIAM J., 167-182. 1, 2.2

[3] H. Y. Alfifi, T. R. Marchant, Feedback Control for a Diffusive Delay Logistic Equation: Semi-analytical Solutions, IAENG Int. J. Appl. Math., 48 (2018), 317-323. 1, 2.2

[4] H. Y. Alfifi, T. R. Marchant, M. I. Nelson, Generalised diffusive delay logistic equations: semi-analytical solutions, Dyn. Contin. Discrete Impuls. Syst. Ser. B Appl. Algorithms, 19 (2012), 579-596. 1, 2.1, 2.2

[5] H. Y. Alfifi, T. R. Marchant, M. I. Nelson, Semi-analytical solutions for the 1- and 2-D diffusive Nicholson's blowflies equation, IMA J. Appl. Math., 79 (2014), 175-199. 1, 2.1, 2.2, 4.3

[6] H. Y. Alfifi, T. R. Marchant, M. I. Nelson, Non-smooth feedback control for Belousov-Zhabotinskii reaction-diffusion equations: semi-analytical solutions, J. Math Chem., 54 (2016), 1632-1657. 1, 2.1

[7] M. R. Alharthi, T. R. Marchant, M. I. Nelson, Mixed quadratic-cubic autocatalytic reaction-diffusion equations: semianalytical solutions, Appl. Math. Model., 38 (2014), 5160-5173. 1

[8] K. S. Al Noufaey, T. R. Marchant, M. P. Edwards, The diffusive Lotka-Volterra predator-prey system with delay, Math. Biosci, 270 (2015), 30-40. 1

[9] N. C. Chi, E. AvilaVales, G. Garcia Almeida, Analysis of a HBV model with diffusion and time delay, J. Appl. Math., 2012 (2012), 25 pages. 1

[10] T. Erneux, Applied Delay Differential Equations, Springer, New York, (2009). 4.1

[11] C. A. J. Fletcher, Computational Galerkin Methods, Springer-Verlag, New York, (1984). 2.2

[12] J. Hale, Theory of Functional Differential Equations, Springer-Verlag, New York-Heidelberg, (1977). 4.1

[13] K. Hattaf, N. Yousfi, Global dynamics of a delay reaction-diffusion model for viral infection with specific functional response, Comput. Appl. Math., 34 (2015), 807-818. 1

[14] K. Hattaf, N. Yousfi, A numerical method for delayed partial differential equations describing infectious diseases, Comput. Math. Appl., 72 (2016), 2741-2750. 1

[15] E. V. Koonin, T. G. Senkevich, V. V. Dolja, The ancient Virus World and evolution of cells, Biol. Direct, 1 (2006), 1-27. 1

[16] K. Manna, A non-standard finite difference scheme for a diffusive HBV infection model with capsids and time delay, J. Difference Equ. Appl., 23 (2017), 1901-1911. 1

[17] T. R. Marchant, Cubic autocatalytic reaction diffusion equations: semi-analytical solutions, R. Soc. Lond. Proc. Ser. A Math. Phys. Eng. Sci., 458 (2002), 873-888. 2.2, 2.2

[18] T. R. Marchant, M. I. Nelson, Semi-analytical solution for one-and two-dimensional pellet problems, Proc. R. Soc. Lond., 460 (2004), 2381-2394. 1, 2.1, 2.2

[19] H. Miao, Z. D. Teng, X. Abdurahman, Z. M. Li, Global stability of a diffusive and delayed virus infection model with general incidence function and adaptive immune response, Comp. Appl. Math., 37 (2018), 3780-3805. 1

[20] M. A. Nowak, S. Bonhoeffer, A. M. Hill, R. Boehme, H. C. Thomas, H. McDade, Viral dynamics in hepatitis B virus infection, Proc. Natl. Acad. Sci., 93 (1996), 4398-4402. 1

[21] M. H. Qiao, H. Qi, Dynamics of the HBV Model with Diffusion and Time Delay, IEEE International Workshop on Chaos-Fractals Theories and Applications, 2009 (2009), 297-300. 1

[22] G. D. Smith, Numerical Solution of Partial Differential Equations: Finite Difference Methods, Oxford University Press, New York, (1985). 4.1

[23] M. P. Taylor, O. Kobiler, L. W. Enquist, Alphaherpesvirus axon-to-cell spread involves limited virion transmission, Proc. Natl. Acad. Sci., 109 (2012), 17046-17051. 1

[24] K. F. Wang, W. D. Wang, Propagation of HBV with spatial dependence, Math. Biosci., 210 (2007), 78-95. 1

[25] K. F. Wang, W. D. Wang, S. P. Song, Dynamics of an HBV model with diffusion and delay, J. Theoret. Biol., 235 (2008), 36-44. 1

[26] R. Xu, Z. Ma, An HBV model with diffusion and time delay, J. Theoret. Biol., 257 (2009), 499-509. 1

[27] K. Zhuang, Spatiotemporal Dynamics of a Delayed and Diffusive Viral Infection Model with Logistic Growth, Math. Comput. Appl., 22 (2017), 19 pages. 1, 4.2 